\title{
SIDELOADING - INGESTION OF LARGE POINT CLOUDS INTO THE APACHE SPARK BIG DATA ENGINE
}

\author{
J. Boehm, K. Liu, C. Alis \\ Dept. of Civil, Environmental and Geomatic Engineering, University College London, UK \\ j.boehm@ucl.ac.uk
}

Commission II, WG II/5

KEY WORDS: Big Data, LiDAR, Cloud Computing, Point Cloud, Spark

\begin{abstract}
:
In the geospatial domain we have now reached the point where data volumes we handle have clearly grown beyond the capacity of most desktop computers. This is particularly true in the area of point cloud processing. It is therefore naturally lucrative to explore established big data frameworks for big geospatial data. The very first hurdle is the import of geospatial data into big data frameworks, commonly referred to as data ingestion. Geospatial data is typically encoded in specialised binary file formats, which are not naturally supported by the existing big data frameworks. Instead such file formats are supported by software libraries that are restricted to single CPU execution. We present an approach that allows the use of existing point cloud file format libraries on the Apache Spark big data framework. We demonstrate the ingestion of large volumes of point cloud data into a compute cluster. The approach uses a map function to distribute the data ingestion across the nodes of a cluster. We test the capabilities of the proposed method to load billions of points into a commodity hardware compute cluster and we discuss the implications on scalability and performance. The performance is benchmarked against an existing native Apache Spark data import implementation.
\end{abstract}

\section{INTRODUCTION}

While the origins of the term "Big Data" itself might be complex (Diebold, 2012) and disputed, one of the most commonly accepted definitions of the term was given by Laney (2001). He observes "data management challenges along three dimensions: volume, velocity and variety". These are often referred to as the 3 Vs. While his observations were made in the particular context of e-commerce they were subsequently generalized. Nowadays the term spans several disciplines and the phenomenon continues to grow, so that some even see it as an emerging discipline. The phenomenon is not unknown to the geospatial community and big spatial data has now been identified as an emerging research trend (Eldawy and Mokbel, 2015a). We will focus on a special area of big spatial data and a particular challenge in data management. We will focus on point clouds and their volume.

\subsection{Point Cloud Data Use}

In the geospatial domain we have now reached the point where data volumes we handle have clearly grown beyond the capacity of a single desktop computer. This is particularly true in the area of point clouds. Examples are the massive point cloud data sets of national mapping or environmental agencies that were released to the public domain in recent years. One of these data sets is the Dutch AHN2 which contains about 400 billion points (Swart, 2010). Another example is the 11 terabyte of LiDAR data the UK's Environment Agency has released through their Geomatics LIDAR portal (Whitworth, 2015).

The phenomenon of big LiDAR data sets is however not constraint to national agencies, but is observed throughout the industry. The preliminary results from the recent OGC survey regarding the current state of standards and best practices in the management of point cloud data, have shown that many users today have to handle very large point clouds (see Figure 1). The majority of users have handled point clouds with more than 1 billion points over the last 12 months. Twenty percent of the users have handled more than 1 trillion points over the same period. This figures clearly show that point cloud data storage clearly faces the challenge of volume, one of the $3 \mathrm{Vs}$ and puts it firmly in the domain of big data.

The storage mechanisms typically employed for point cloud storage in industry on the other hand do not reflect this, as shown in Figure 2. (Multiple answers were possible so the sum of all answers is more than $100 \%$ ). Most of the respondents store the data in files on a traditional file system and very few use cloud technology, a typical big data tool.

\subsection{Geo Data as Big Data}

The huge data volume and the underdeveloped adoption of big data tools, both clearly indicate a gap in the current toolchain of big point cloud data management. These observations confirm the need to develop or adapt cloud computing techniques that are appropriate for large collections of point cloud data. This is a conclusion already drawn by Eldawy \& Mokbel (2015a) in the general context of GIS data.

The EU FP7 project IQmulus is an initiative to provide a highvolume fusion and analysis platform for geospatial point clouds, coverages and volumetric data (IQmulus, 2012). It attempts to leverage the information hidden in large heterogeneous geospatial data sets and make them a practical choice to support reliable decision making.

The experiments conducted for this work are part of this effort to provide scalable, distributed and cloud based tools to handle big geospatial data. We concentrate here on point clouds as we see them as a premier example for the growing data volume. For this work we also focus on the first step in the Big Data pipeline, the import of data into the framework, commonly referred to as data ingestion. 


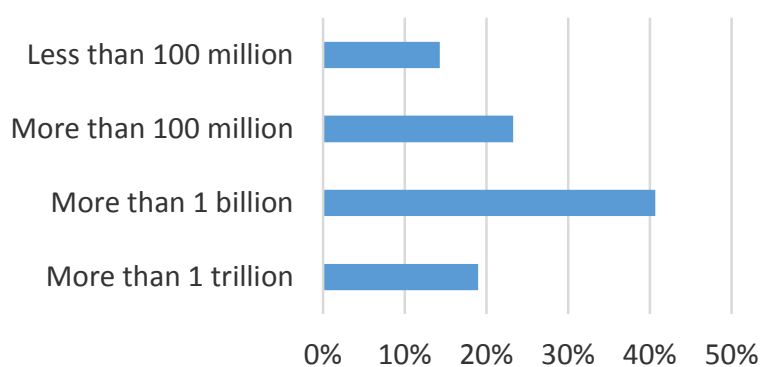

Figure 1: Typical number of points for point clouds encountered by users in practice. Preliminary results extracted from user responses to the OGC survey regarding the current state of standards and best practices in the management of point cloud data.

\subsection{Cloud Compute Engines}

While the formal definition of big data precedes it, many will have become aware of cloud computing through the publication of Google's MapReduce approach (Dean and Ghemawat, 2008). MapReduce is a programming paradigm to process large dataset in a distributed fashion on a commodity hardware cluster. Apache Hadoop (Hadoop, 2016) is an open-source implementation of this paradigm and more generally a family of big data tools. This includes the Hadoop Distributed File System (HDFS), which is a scalable and resilient cluster files system (Shvachko et al., 2010). There already exist a small number of geospatial frameworks built on Hadoop, e.g. SpatialHadoop (Eldawy and Mokbel, 2015b).

In 2010 Spark was developed as a response to some shortcomings in Hadoop. It was particularly designed to accommodate applications that "reuse a working set of data across multiple parallel operations" (Zaharia et al., 2010). Machine learning applications were explicitly targeted as falling into this category. It was shown that Spark can outperform Hadoop by ten times in an iterative machine learning job.

Apache Spark heavily relies on the concept of Resilient Distributed Datasets (RDDs) (Zaharia et al., 2012). They allow the in-memory distribution of large datasets across a cluster.

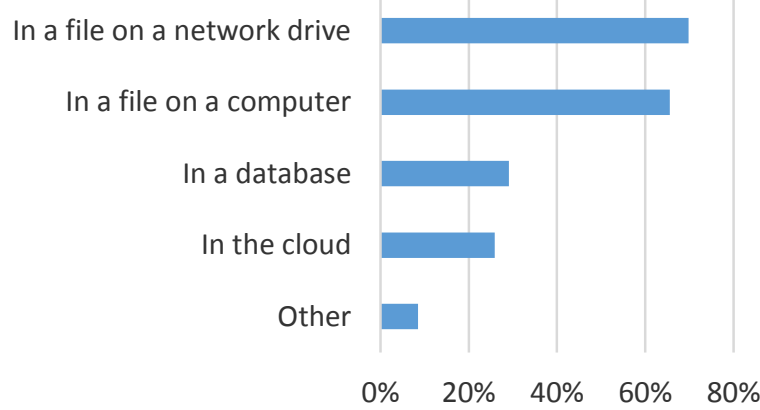

Figure 2: Preliminary results on the typical storage mechanisms for point clouds of the OGC survey regarding the current state of standards and best practices in the management of point cloud data.
They are resilient to node failures as the data is replicated across the cluster. Keeping the data in memory rather than on disk allows for the high speed in computation.

Again a small number of geospatial frameworks exist that leverage the capabilities of Apache Spark. SpatialSpark was developed for efficient spatial join queries (You et al., 2015). GeoTrellis is focusing on raster data (Kini and Emanuele, 2014).

\section{RELATED WORK}

\subsection{IQmulus Architecture}

The IQmulus project has suggested and implemented a distributed architecture dedicated for large geospatial data processing (Krämer and Senner, 2015). While our proposed framework is based on Apache Spark and therefore does not rely on the scheduling components of the IQmulus architecture, it is still relevant as we use its storage mechanism. The IQmulus architecture uses HDFS (see above) as a mature and scalable storage mechanism.

In particular, it uses a NFS wrapper which provides a mountable file system. This makes HDFS indirectly available to the user processes through the operating system's file system. One intention of this work is to explore the suspected implications in file access and scalability of this design. Our approach makes heavy use of the fact that the data stored in HDFS is available via the standard filesystem API.

\subsection{Spark SQL IQmulus Library}

The Spark SQL IQmulus library (Brédif, 2015) implements in Scala a Spark DataFrame reader/writer interface for common LiDAR file formats, such as LAS and PLY. The use of this library has already been demonstrated in an implementation of a scalable rendering pipeline for large point clouds (Brédif et al., 2015). The advantage of this library lies in the fact that it implements the import of strongly formatted data directly within the Spark framework. Hence it can make use of the high level abstraction of Data Sources available in Spark. These include the local file system, and distributed filesystems such as HDFS.

The disadvantage of this high level abstraction is that existing format libraries can typically not be used as they assume a classic filesystem interface. This means that the format reader has to be re-implement from scratch. This can cumbersome if a full set of features is required. It might even be prohibitive in cases where the format description is not publicly available, e.g. for proprietary formats.

\subsection{Single CPU libraries}

As expected for a matured data source such as point clouds various libraries exist for reading the most popular file formats. We focus on the ASPRS LAS format ("LAS Specification Version 1.3," 2009) as the most commonly used LiDAR format. The available libraries for LAS differ in their support for the various features of the format such as coordinate reference systems, variable length records and waveform data. The LASlib from the LAStools set of LiDAR processing tools (Isenburg and Schewchuck, 2007) is a popular option which implements many of the features. 
The liblas library (Butler et al., 2011, 2011) is another C/C++ library for reading and writing LAS files. It also provides a Python interface. The source code of liblas is available under the terms of the BSD license. It assumes a local filesystem API for file access and allows reading a LAS file record by record.

Laspy is a pure Python implementation for the LAS format (Brown, 2012). It uses memory-mapped files to accelerate access. While this has significant performance advantages in some cases we have also experienced problematic behaviour with this library in a cluster environment.

\section{PROPOSED METHOD}

The idea behind the proposed method is to re-use existing file format libraries for the data import. The typically use a classic filesystem interface. If we have the distributed file system (DFS) mounted as a virtual filesystem (VFS) in the node's operating system, every node can access the files and load them via a filesystem interface. We therefore only need to distribute the filenames to the individual nodes to perform the task in parallel. Figure 3 schematically shows how the approach bypasses the direct data import and uses the VFS for the actual file access. We therefore refer to the method as sideloading. We use Python to implementing the distribution via a mapping function as it is one of the support APIs of Apache Spark and also supports many existing file format libraries.

\subsection{Naïve Sideloading}

Sideloading can most easily be implemented as a mapping function, which maps a list of filenames to their data content. In the case of point cloud import from LAS files this requires a mapping function that maps LAS file names to lists of XYZ coordinates:

$$
\text { ["file1.las", "file2.las", ... }] \rightarrow[(x, y, z, \ldots),(x, y, z, \ldots), \ldots]
$$

If we use Spark's flatMap capability, we can effectively create mapping function that creates a single list of XYZ coordinates from all input point clouds:

$$
\text { ["file1.las", "file2.las", ...] } \rightarrow[x, y, z, \ldots, x, y, z, \ldots]
$$

The pseudo code notation in Figure 4 shows how this can easily be achieved in Spark. A mapping function load_map uses an
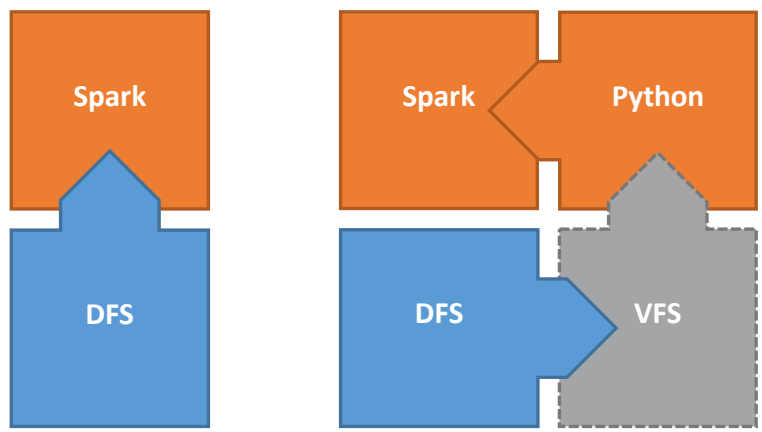

Figure 3: comparing the schematics of direct data import into Sark from a Distributed File System (DFS) versus sideloading via a Virtual local File System (VFS) and Python.

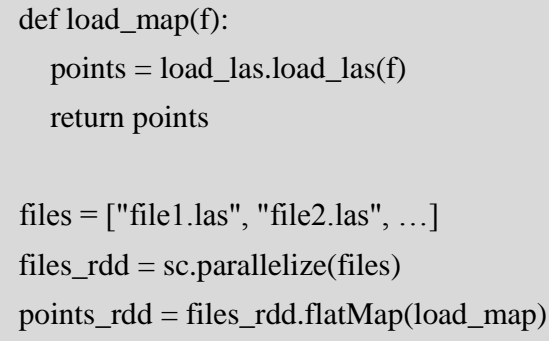

Figure 4: Pseudo code of a naïve mapping function for sideloading LiDAR data.

existing single CPU LAS library to load an individual LAS file. Spark's resilient distributed dataset is created form a list of input files. The mapping function is evoked on the RDD and the contents of the files are mapped to a single new RDD.

\subsection{Slicing}

The naïve mapping function introduced above has two major problems. First it maps files to the individual nodes / workers. Therefore the number of files have to be sufficiently larger than the number of nodes / workers. If not there will be nodes that remain idle as they have no file to work on. Secondly each file is read in its entirety into the worker node's memory. If however the file is larger than the available memory this can cause the mapping function to fail.

It is therefore an obvious idea to subdivide each file further into slices. Instead of loading entire files only slices of each file ae loaded. This means instead of distributing files to nodes / workers, slices are distributed. Subsequently many more tasks are created which leads to a finer granularity for the workload distribution. This should be generally advantageous for distributed computing.

The approach can be achieved in two simple intermediate mapping functions, which store the point count for each file and generate the slices consisting of a start- and end-point in the files data records.

["file1.las", "file2.las", ... ]

$\rightarrow$ [("file1.las", point count), ("file2.las",point count), ... ]

[("file1.las", point count), ("file2.las", point count), ... ] $\rightarrow$ [("file1.las", start1, end1), ("file1.las", start2, end2 ), ... ]

\section{TEST SCENARIO}

The following section briefly describe the test scenario consisting of the data and hardware that is used to obtain the running times and other characteristics of the proposed method.

\subsection{Data Sets}

Two datasets a re selected for the experimentation. The first is a medium size dataset. It consists of 99 files and holds a combined 1.7 billion $\left(10^{9}\right)$ points. It is a mobile mapping data set of the Bloomsbury area around University College London. It has an average point spacing of better than $20 \mathrm{~mm}$. Figure 5 gives an overview of the coverage of the data set. Figure 6 gives a detailed view and shows the density of the data set. 


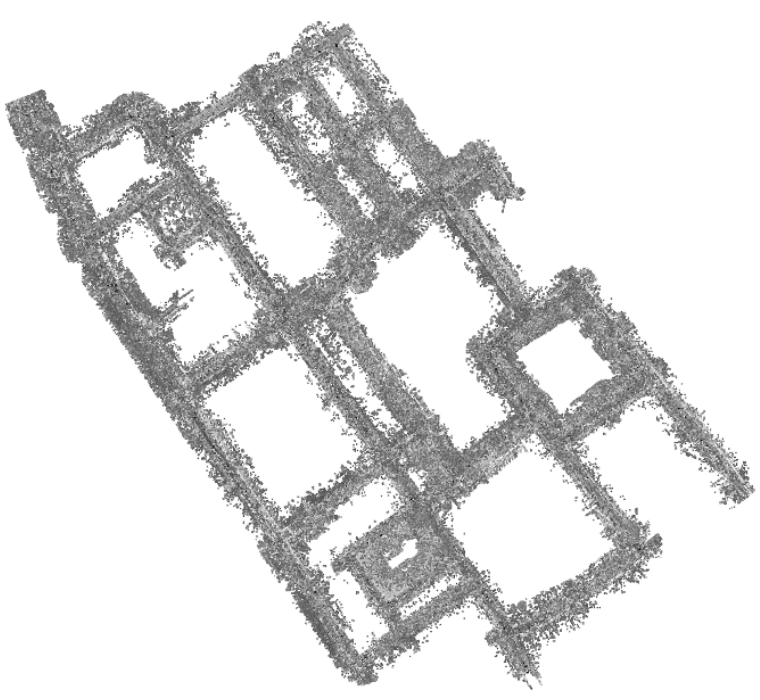

Figure 5: Overview of the medium size data set used in the experiment.

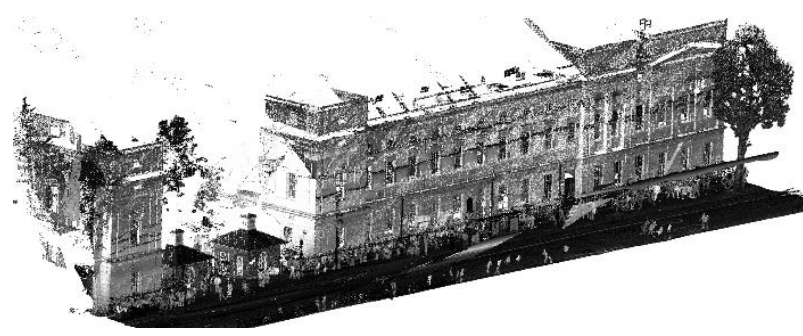

Figure 6: Detail view of the medium size data set used in the experiment.

The second dataset is a larger sized LiDAR point cloud. It is a classical airborne LiDAR dataset storing multiple returns. It has an average density of 1 point per square meter. The dataset is provided by CNR-IMATI. It covers the coastal area of Regione Liguria. Figure 7 shows a detail of the dataset.

\subsection{Cluster Architecture}

The cluster we use to establish performance metrics is provided by Fraunhofer IGD Darmstadt. It consists of 1 master node and 5 worker nodes. Each worker node has four virtual cores, which are occupied by 4 workers. So overall we have 20 workers with a combined memory of $34 \mathrm{~GB}$. The prototype implementation is

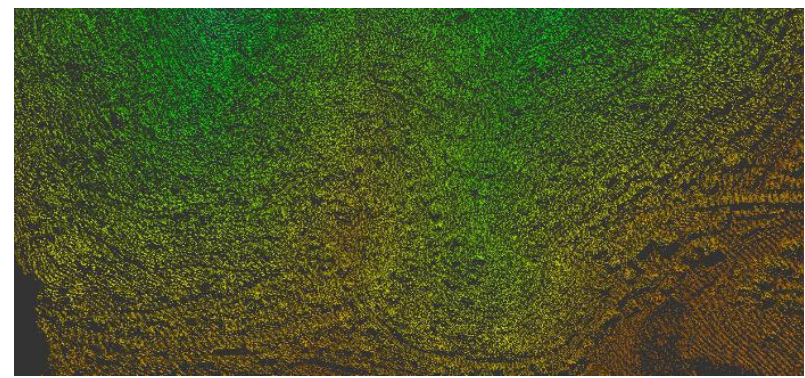

Figure 7: Detail of the large size data set used in the experiment. based on Apache Spark 1.6 which is run in cluster mode. We use Python to implement the mapping functions and liblas for the LAS file handling.

\section{EXPERIMENTAL RESULTS}

The following sections show the experimental results obtained using the setup described above.

\subsection{Medium Dataset}

The first experiments explore the effect of varying the number of nodes while keeping the data volume fixed. We use a subset of the medium sized dataset, consisting of 10 files holding 180 million points. We compare naïve sideloading to sideloading with slicing. Both are benchmarked against the native Spark implementation of a data import provided by the Spark SQL IQmulus library. The results of this experiment are shown in Figure 8 . We can see that naïve sideloading does not scale very well over an increasing number of nodes. This is expected as we have observed above that the number of files must be sufficiently larger than the umber of nodes / workers. With only 10 files and a maximum of 20 workers this is clearly not the case. When we introduce slicing however we can observe almost perfect scaling of the runtime over the number of nodes. We can observe the same for the native Spark implementation.

The second set of experiments explore the behaviour over an increase in the data volume. We scale the data import from a few million to just under 2 billion points. Figure 9 shows the graphical results. Again we can see that naïve sideloading does not perform efficient over a smaller subset of the data. It also shows an offset in performance overall. Sideloading with slicing on the other hand scales perfectly linear with the increase in data volume, which is the desired behaviour. The native Spark implementation seems to perform best on the maximum data size. The results of the experiment can also be interpreted as data throughput, i.e. millions of points ingested per second. Figure 10 shows the bar graph for data throughput. While sideloading with slicing seems to perform at par with a native Spark data import in most cases, for the largest data size that does not hold.

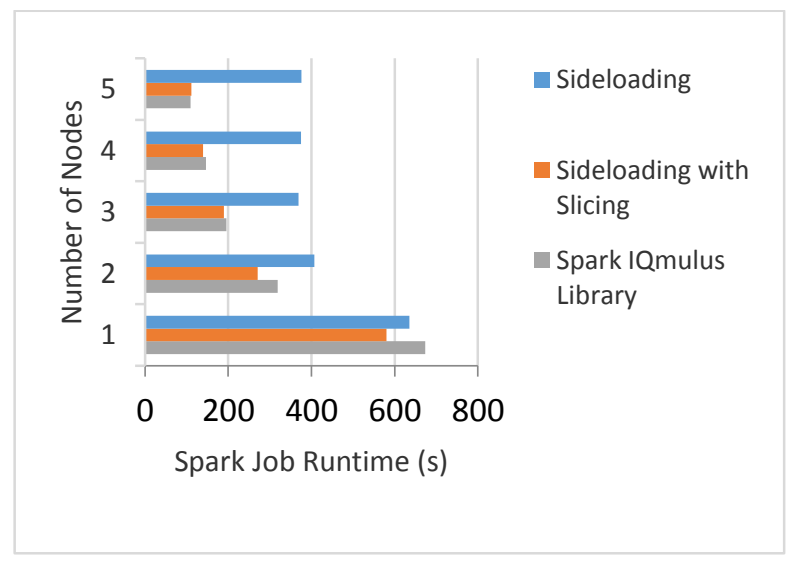

Figure 8: Effect of varying the number of nodes for a fixed sized point cloud. 


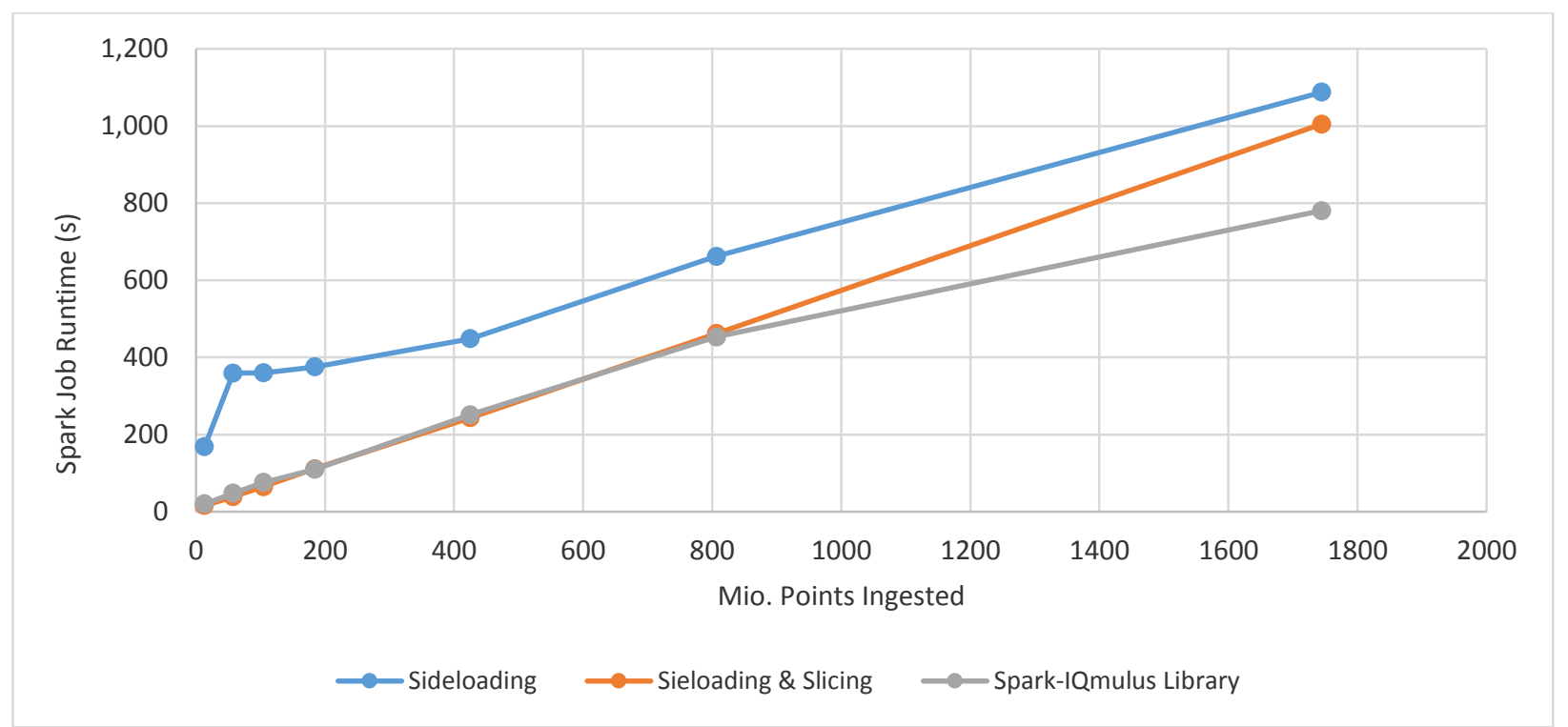

Figure 9: Runtime for sideloading over varying number of points from a medium sized point cloud data set.

\subsection{Large Dataset}

The performance penalty becomes more apparent when the data volume is further increased. Figure 11 shows the results on the larger dataset with a maximum of just under 8 billion points. Again sideloading with slicing performs perfectly linear under the increase of data volume. However it is apparent that there is a performance penalty over a native Spark data import.

\section{CONCUISONS \& OUTLOOK}

We have shown a simple yet efficient method of ingesting very large point clouds into the Apache Spark Big Data Engine. The proposed method re-uses existing single CPU libraries for file format interpretation. It therefore requires minimal implementation effort and is easy to adapt to various file formats including proprietary formats. When we introduce slicing it

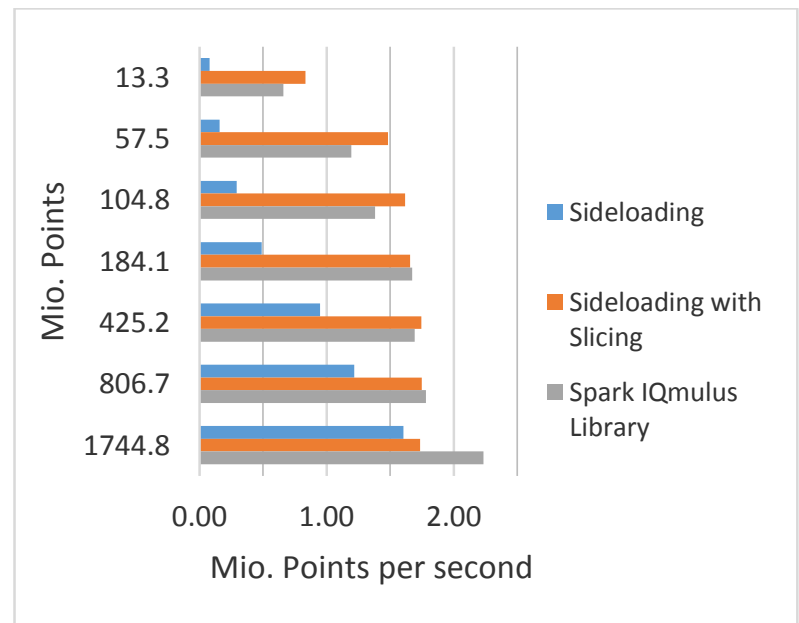

Figure 10: Throughput of a five node cluster with different point cloud sizes. scales linear both with a varying node count and varying data volume. There is an overall performance penalty when compared to a native Spark data import implementation. We recon that for most applications this is acceptable in the data ingestion stage.

Understanding large point clouds as big data and using an established big data toolchain opens many possibilities. We have already shown the successful use of cloud based machine learning for point cloud classification (Liu and Boehm, 2015). While the established tools do not typically provide spatial functionality such as indexing an query, these can be implemented on top of existing frameworks (Alis et al., 2016).

\section{ACKNOWLEDGEMENTS}

We would like to acknowledge that this work is in part supported by EU grant FP7-ICT-2011-318787 (IQmulus). We also acknowledge that parts of this work are supported by an Amazon AWS in Education Research Grant award.

\section{REFERENCES}

Alis, C., Boehm, J., Kun, L., 2016. Parallel Processing of Big Point Clouds using Z-Order-Based Partitioning. Int. Arch. Photogramm. Remote Sens. Spat. Inf. Sci. XLI-B3.

Brédif, M., 2015. Spark SQL IQmulus Library [WWW Document]. URL https://github.com/IGNF/spark-iqmulus

Brédif, M., Vallet, B., Ferrand, B., 2015. Distributed Dimensionality-Based Rendering of LIDAR Point Clouds. ISPRS - Int. Arch. Photogramm. Remote Sens. Spat. Inf. Sci. XL3/W3, 559-564. doi:10.5194/isprsarchives-XL-3-W3-559-2015

Brown, G., 2012. Laspy [WWW Document]. URL https://github.com/grantbrown/laspy

Butler, H., Loskot, M., Vachon, P., Vales, M., Warmerdam, F., 2011. libLAS: ASPRS LAS LiDAR Data Toolkit. Last Access 15. 


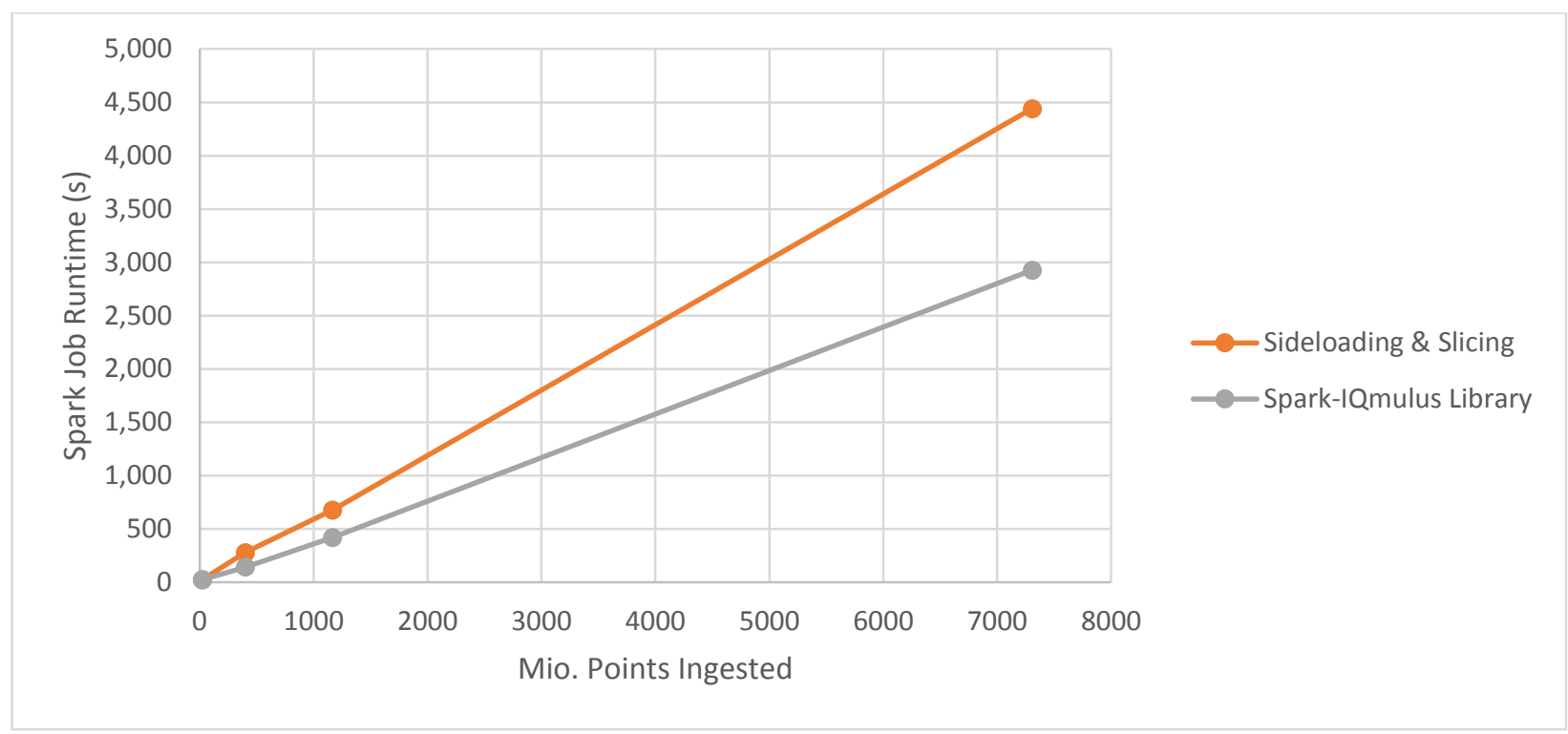

Figure 11: Runtime for sideloading of a large sized point cloud data set over varying number of points.

Dean, J., Ghemawat, S., 2008. MapReduce: simplified data processing on large clusters. Commun. ACM 51, 107-113.

Diebold, F.X., 2012. A Personal Perspective on the Origin (s) and Development of'Big Data': The Phenomenon, the Term, and the Discipline, Second Version.

Eldawy, A., Mokbel, M.F., 2015a. The Era of Big Spatial Data, in: Proceedings of the International Workshop of Cloud Data Management CloudDM 2015. Seoul, Korea.

Eldawy, A., Mokbel, M.F., 2015b. SpatialHadoop: A MapReduce framework for spatial data, in: Data Engineering (ICDE), 2015 IEEE 31st International Conference on. IEEE, pp. $1352-1363$.

Hadoop [WWW Document], 2016. URL http://hadoop.apache.org/ (accessed 4.1.16).

IQmulus [WWW Document], 2012. URL http://iqmulus.eu/

Isenburg, M., Schewchuck, J., 2007. LAStools: converting, viewing, and compressing LIDAR data in LAS format. Avaliable Httpwww Cs Unc Edu Isenburglastools.

Kini, A., Emanuele, R., 2014. Geotrellis: Adding geospatial capabilities to spark. Spark Summit.

Krämer, M., Senner, I., 2015. A modular software architecture for processing of big geospatial data in the cloud. Comput. Graph. 49, 69-81. doi:10.1016/j.cag.2015.02.005

Laney, D., 2001. 3D data management: Controlling data volume, velocity and variety. META Group Res. Note 6, 70.

LAS Specification Version 1.3, 2009.

Liu, K., Boehm, J., 2015. Classification of Big Point Cloud Data Using Cloud Computing. Int. Arch. Photogramm. Remote Sens. Spat. Inf. Sci. 40, 553.

Shvachko, K., Kuang, H., Radia, S., Chansler, R., 2010. The hadoop distributed file system, in: Mass Storage Systems and Technologies (MSST), 2010 IEEE 26th Symposium on. IEEE, pp. 1-10.
Swart, L.T., 2010. How the Up-to-date Height Model of the Netherlands (AHN) became a massive point data cloud. NCG KNAW 17.

Whitworth, M., 2015. Laser surveys light up open data [WWW Document]. URL https://environmentagency.blog.gov.uk/2015/09/18/lasersurveys-light-up-open-data/ (accessed 4.1.16).

You, S., Zhang, J., Gruenwald, L., 2015. Large-scale spatial join query processing in cloud, in: Data Engineering Workshops (ICDEW), 2015 31st IEEE International Conference on. IEEE, pp. 34-41.

Zaharia, M., Chowdhury, M., Das, T., Dave, A., Ma, J., McCauley, M., Franklin, M.J., Shenker, S., Stoica, I., 2012. Resilient distributed datasets: A fault-tolerant abstraction for inmemory cluster computing, in: Proceedings of the 9th USENIX Conference on Networked Systems Design and Implementation. USENIX Association, pp. 2-2.

Zaharia, M., Chowdhury, M., Franklin, M.J., Shenker, S., Stoica, I., 2010. Spark: Cluster Computing with Working Sets. HotCloud $10,10-10$. 